\title{
Global Existence and Energy Decay Rates for a Kirchhoff-Type Wave Equation with Nonlinear Dissipation
}

\author{
Daewook Kim, ${ }^{1}$ Dojin Kim, ${ }^{2}$ Keum-Shik Hong, ${ }^{3}$ and Il Hyo Jung ${ }^{1}$ \\ ${ }^{1}$ Department of Mathematics, Pusan National University, 30 Jangjeon-dong, Geumjeong-gu, Busan 609-735, Republic of Korea \\ ${ }^{2}$ Department of Mathematics, Oregon State University, Corvallis, OR 97331, USA \\ ${ }^{3}$ Department of Cogno-Mechatronics Engineering and School of Mechanical Engineering, Pusan National University, \\ Busan 609-735, Republic of Korea
}

Correspondence should be addressed to Il Hyo Jung; ilhjung@pusan.ac.kr

Received 23 January 2014; Accepted 23 February 2014; Published 7 April 2014

Academic Editors: D. Baleanu and H. Jafari

Copyright (C) 2014 Daewook Kim et al. This is an open access article distributed under the Creative Commons Attribution License, which permits unrestricted use, distribution, and reproduction in any medium, provided the original work is properly cited.

The first objective of this paper is to prove the existence and uniqueness of global solutions for a Kirchhoff-type wave equation with nonlinear dissipation of the form $K u^{\prime \prime}+M\left(\left|A^{1 / 2} u\right|^{2}\right) A u+g\left(u^{\prime}\right)=0$ under suitable assumptions on $K, A, M(\cdot)$, and $g(\cdot)$. Next, we derive decay estimates of the energy under some growth conditions on the nonlinear dissipation $g$. Lastly, numerical simulations in order to verify the analytical results are given.

\section{Introduction}

A mathematical model for the transverse deflection of an elastic string of length $L>0$ whose ends are held a fixed distance apart is written in the form of the hyperbolic equation

$$
\frac{\partial^{2} u(x, t)}{\partial t^{2}}-\left(\alpha+\beta \int_{0}^{L}\left|\frac{\partial u(x, t)}{\partial x}\right|^{2} d x\right) \frac{\partial^{2} u(x, t)}{\partial x^{2}}=0
$$

which was proposed by Kirchhoff [1], where $u(x, t)$ is the deflection of the point $x$ of the string at the time $t$ and $\alpha>0, \beta$ are constants. Kirchhoff first introduced (1) in the study of the oscillations of stretched strings and plates, so that (1) is called the wave equation of Kirchhoff type. The Kirchhofftype model also appeared in scientific research for beam or plate [2-5]. Such nonlinear Kirchhoff model gives one way to describe the dynamics of an axially moving string. In recent years, axially moving string-like continua such as wires, belts, chains, and band saws have been the subject of study of researchers [6-14].
The mathematical aspects of the natural generalization of the model (1) in $\Omega \subset \mathbb{R}^{n}$ :

$$
\begin{gathered}
u^{\prime \prime}-M\left(\int_{\Omega}|\nabla u|^{2} d x\right) \Delta u+g\left(u^{\prime}\right)=0, \\
u(0)=u_{0}, \quad u^{\prime}(0)=u_{1},
\end{gathered}
$$

under some assumptions on $M(\cdot), g(\cdot)$, have been studied, using different methods, by many authors $[6,8,15-22]$.

When $g(\cdot)=0$ and $n=1$, the problem (2)-(3) was studied by Dickey [16] and Bernstein [15] who considered analytic functions as the initial data (see also Yamada [21] and Ebihara et al. [17]). In case when $g(\cdot)=0$ and $n \geq 1$, Pohožaev [22] obtained the existence and uniqueness of global solutions for the problem (2)-(3). Lions [20] also formulated Pohožaev's results in an abstract context and obtained better results.

Equation (2) with linear dissipative term, that is, $g\left(u^{\prime}\right)=$ $\delta u^{\prime}(\delta>0)$, was investigated by Mizumachi [23], Nishihara and Yamada [24], Park et al. [25], and Jung and Choi [26]. In fact, they studied the existence, uniqueness, and the energy decay rates of solutions for the problem (2)-(3). On the other hand, related works to a Kirchhoff-type equation with $K u^{\prime \prime}$ instead of $u^{\prime \prime}$ can be found in Levine [19]. Jung and Lee [27] got the result for a Kirchhoff-type equation with strong 
dissipative term. But they studied a simple form with the coefficient $M(\cdot) \equiv 1$. In case of the equation concerning nonlinear Kirchhoff-type coefficient, recently, Kim et al. [8], Ghisi and Gobbino [28], and Aassila and Kaya [29] have studied existence and energy decay rates of global (or local) solutions for the equation. By giving some suitable smallness conditions on the sizes of the initial data, they assured global existence and energy decay rates for the solutions.

In this paper, we study the existence, uniqueness, and the decay estimates of the energy for a class of Kirchhoff-type wave equations in a Hilbert space $H$ :

$$
\begin{gathered}
K u^{\prime \prime}+M\left(\left|A^{1 / 2} u\right|^{2}\right) A u+g\left(u^{\prime}\right)=0 \quad \text { in } H, \\
u(0)=u_{0}, \quad\left(K u^{\prime}\right)(0)=K^{1 / 2} u_{1},
\end{gathered}
$$

where $K$ and $A$ are linear operators in $H$ and $M(\cdot) \in C^{1}[0, \infty)$. For global existence of this problem, we give some suitable smallness conditions. So, the main contribution of these results is to consider a general model which contains the concrete model (2)-(3) and to improve the results of KouémouPatcheu [30] and Jung and Choi [26]. Moreover, as an application, we give some simulation results about solution's shapes and the algebraic decay rate for a Kirchhoff-type wave equation with nonlinear dissipation.

The method applied in this paper is based on the multipliers technique [31], Galerkin's approximate method, and some integral inequalities due to Haraux [32].

This paper is organized as follows. In Section 2, we recall the notation, hypotheses, and some necessary preliminaries and prove the existence and uniqueness of global solutions for the system (4) by employing Feado-Galerkin's techniques under suitable smallness condition. In Section 3, we derive the energy decay rates by using the multiplier technique under suitable growth conditions on $g$. Finally, in Section 4, we give an example and its numerical simulations to illustrate our results.

\section{Preliminaries and Existence}

Let $\Omega$ be a bounded open domain in $\mathbb{R}^{n}$ having a smooth boundary $\Gamma$ and $H=L^{2}(\Omega)$ with inner product and norm denoted by $(\cdot, \cdot)$ and $|\cdot|$, respectively. Let $K$ be a linear, positive, and self-adjoint operator on $H$; that is, there is a constant $c>0$ such that

$$
(K u, u) \geq c|u|^{2}, \quad \forall u \in H .
$$

Let $A$ be a linear, self-adjoint, and positive operator in $H$, with domain $V:=D(A)$ dense in $H, K A=A K$ on $D(A) \cap D(K)$, and the graph norm denoted by $\|$. $\|$. We assume that the imbedding $V \subset H$ is compact. Identifying $H$ and its dual $H^{\prime}$, it follows that $V \subset H \subset V^{\prime}$, where $V^{\prime}$ is the dual of $V$. Let $\langle\cdot, \cdot\rangle_{V^{\prime}, V}$ denote the duality pairing between $V^{\prime}$ and $V$ and $W:=D\left(A^{1 / 2}\right)$.

Throughout the paper we will make the following assumptions.

(M) $M(s)$ is a $C^{1}[0, \infty)$ real function and $M^{\prime}(s) \geq$ 0 . Furthermore, there exist some positive constants $\beta$ and $\gamma_{0}$ such that $M(s) \geq \beta>0$ for all $s \geq 0$ and $\left|M^{\prime}(s) s\right| / M(s) \leq \gamma_{0}$.

(G) $g: \mathbb{R} \rightarrow \mathbb{R}$ is a nondecreasing continuous function such that $g(0)=0$ and there is a constant $k>0$ and $q \geq 1$ such that

$$
|g(x)| \leq k\left(1+|x|^{q}\right) \quad \forall x \in \mathbb{R} .
$$

And $(g(u), A u) \geq 0$ for all $u \in D(A) \cap D\left(A^{1 / 2}\right)$. Note that the last assumption of $(\mathrm{G})$ makes sense. In fact, when $A=-\Delta$ and $g(u)=|u|^{\alpha} u, \alpha \geq 1$, we can easily show that $(g(u), A u) \geq 0$ for all $u \in D(A) \cap D\left(A^{1 / 2}\right)$.

(H) $M^{\prime}(s)>M(s)|g(x)|, s \in[0, \infty), x \in \mathbb{R}$.

(S) $V \subset L^{q+1}(\Omega)$ for some $q \geq 1$.

Let $\bar{M}(t)$ and $E(t)$ be defined as follows:

$$
\begin{gathered}
\bar{M}(t)=\int_{0}^{t} M(s) d s \\
E(t)=\frac{1}{2}\left[\left|K^{1 / 2} u^{\prime}\right|^{2}+\bar{M}\left(\left|A^{1 / 2} u\right|^{2}\right)\right] .
\end{gathered}
$$

And also let us consider the functions

$$
\begin{aligned}
& P(t):=\frac{\left|K^{1 / 2} u^{\prime}(t)\right|^{2}}{M\left(\left|A^{1 / 2} u\right|^{2}\right)}+\left|A^{1 / 2} u(t)\right|^{2}, \\
& Q(t):=\frac{\left|K^{1 / 2} A^{1 / 2} u^{\prime}(t)\right|^{2}}{M\left(\left|A^{1 / 2} u\right|^{2}\right)}+|A u(t)|^{2}, \\
& G(t):=\frac{\left|K^{1 / 2} u^{\prime}(t)\right|}{M\left(\left|A^{1 / 2} u\right|^{2}\right)} .
\end{aligned}
$$

Theorem 1. Let the initial conditions $\left(u_{0}, u_{1}\right) \in W \times L^{2 q}(\Omega)$ satisfy the smallness assumption

$$
\left\|M^{\prime}\right\|_{L^{\infty}([0, P(0)])} B\left(u_{0}, u_{1}\right) \sqrt{Q(0)}<\frac{1}{4},
$$

where $B\left(u_{0}, u_{1}\right)=\max \left\{\left|K^{1 / 2} u_{1}\right| / M\left(\left|A^{1 / 2} u_{0}\right|^{2}\right), M\left(\left|A^{1 / 2} u_{0}\right|^{2}\right) /\right.$ $\left.\left.\left(\left(M\left(\left|A^{1 / 2} u_{0}\right|^{2}\right)\right)^{\prime}-g\left(u_{1}\right) M\left(\left|A^{1 / 2} u_{0}\right|^{2}\right)\right)\right) \sqrt{Q(0)}\right\}$. Then there is a unique function $u \in L^{\infty}(0, T ; W) \cap W^{1, \infty}(0, T ; V) \cap W^{2, \infty}(0$, $T ; H)$ such that, for any $T>0$,

$$
\begin{array}{r}
K u^{\prime \prime}+M\left(\left|A^{1 / 2} u\right|^{2}\right) A u+g\left(u^{\prime}\right)=0 \\
\text { in } L^{(q+1) / q}\left(0, T ; V^{\prime}\right), \\
u(0)=u_{0}, \quad\left(K u^{\prime}\right)(0)=K^{1 / 2} u_{1} .
\end{array}
$$

Proof. Assume that, for simplicity, $V$ is separable; then there is a sequence $\left(e^{j}\right)_{j \geq 1}$ consisting of eigenfunctions of the operator $A$ corresponding to positive real eigenvalues $\mu_{j}$ tending to $+\infty$ so that $A e^{j}=\mu_{j} e^{j}, j \geq 1$.

Let us denote by $V_{m}$ the linear hull of $e^{1}, e^{2}, \ldots, e^{m}$. Note that $\left(e^{j}\right)_{j \geq 1}$ is a basis of $H, V$, and $W$ and hence it is dense in $H, V$, and $W$. 
Approximate Solutions. We search for a function $u_{m}(t)=$ $\sum_{j=1}^{m} g_{j m}(t) e^{j}$ such that, for any $v \in V_{m}, u_{m}(t)$ satisfies the approximate equation

$$
\left(K u_{m}^{\prime \prime}(t)+M\left(\left|A^{1 / 2} u_{m}\right|^{2}\right) A u_{m}+g\left(u_{m}^{\prime}\right), v\right)=0
$$

and the initial conditions as the projections of $u_{0}$ and $u_{1}$ over $V_{m}$ satisfy

$$
\begin{aligned}
u_{m}(0) & =u_{0 m}=\sum_{j=1}^{m}\left(u_{0}, e^{j}\right) e^{j} \longrightarrow u_{0} \quad \text { in } W \\
\left(K u_{m}^{\prime}\right)(0) & =K^{1 / 2} u_{1 m} \\
& =\sum_{j=1}^{m}\left(u_{1}, e^{j}\right) e^{j} \longrightarrow K^{1 / 2} u_{1} \quad \text { in } L^{2 q}(\Omega) .
\end{aligned}
$$

For $v=e^{j}, j=1,2, \ldots m$, the system (13)-(15) of ordinary differential equations of variable $t$ has a solution $u_{m}(t)$ in an interval $\left[0, t_{m}\right)$.

Now we obtain a priori estimates for the solution $u_{m}(t)$ and it can also be extended to $[0, T)$ for all $T>0$.

A Priori Estimate I. Let us consider $v=u_{m}^{\prime}$ in (13). Using (7), we have

$$
\begin{aligned}
& \frac{d}{d t}\left(\left|K^{1 / 2} u_{m}^{\prime}(t)\right|^{2}+\bar{M}\left(\left|A^{1 / 2} u_{m}(t)\right|^{2}\right)\right) \\
& \quad+2\left(g\left(u_{m}^{\prime}(t)\right), u_{m}^{\prime}(t)\right)=0 .
\end{aligned}
$$

Integrating (16) over $(0, t), t \leq t_{m}$, and using (8), we have

$$
\begin{aligned}
2 E(0)= & {\left[\left|K^{1 / 2} u_{m}^{\prime}(t)\right|^{2}+\bar{M}\left(\left|A^{1 / 2} u_{m}(t)\right|^{2}\right)\right] } \\
& +2 \int_{0}^{t}\left(g\left(u_{m}^{\prime}(s)\right), u_{m}^{\prime}(s)\right) d s .
\end{aligned}
$$

Using (5) and (7), we deduce that

$$
\begin{aligned}
2 E(0) \geq & \left|K^{1 / 2} u_{m}^{\prime}(t)\right|^{2}+\beta\left|A^{1 / 2} u_{m}(t)\right|^{2} \\
& +2 \int_{0}^{t} \int_{\Omega} u_{m}^{\prime}(s) g\left(u_{m}^{\prime}(s)\right) d x d s
\end{aligned}
$$

where the left-hand side is constant independent of $m$ and $t$. Thus estimation (18) yields, for any $0<T<\infty$,

$$
\begin{gathered}
u_{m}^{\prime} \text { bounded in } L^{\infty}(0, T ; H), \\
K^{1 / 2} u_{m}^{\prime} \text { bounded in } L^{\infty}(0, T ; H), \\
A^{1 / 2} u_{m} \text { bounded in } L^{\infty}(0, T ; H), \\
u_{m}^{\prime} g\left(u_{m}^{\prime}\right) \text { bounded in } L^{1}([0, T] \times \Omega) .
\end{gathered}
$$

Now we show that $u_{m}(t)$ can be extended to $[0, \infty)$. We need the following smallness assumption:

$$
\begin{aligned}
& \left\|M^{\prime}\right\|_{L^{\infty}([0, P(0)])} \\
& \times \max \left\{\frac{\left|K^{1 / 2} u_{m 1}\right|}{M\left(\left|A^{1 / 2} u_{m 0}\right|^{2}\right)},\left(M\left(\left|A^{1 / 2} u_{m 0}\right|^{2}\right)\right)\right. \\
& \times\left(\left(M\left(\left|A^{1 / 2} u_{m 0}\right|^{2}\right)\right)^{\prime}-g\left(u_{m 1}\right)\right. \\
& \left.\left.\times M\left(\left|A^{1 / 2} u_{m 0}\right|^{2}\right)\right)^{-1} \sqrt{Q(0)}\right\} \\
& \times \sqrt{Q(0)}<\frac{1}{4},
\end{aligned}
$$

where $P(0)=\left(\left|K^{1 / 2} u_{m 1}\right|^{2} / M\left(\left|A^{1 / 2} u_{m 0}\right|^{2}\right)\right)+\left|A^{1 / 2} u_{m 0}\right|^{2}, Q(0)=$ $\left(\left|K^{1 / 2} A^{1 / 2} u_{m 1}\right|^{2} / M\left(\left|A^{1 / 2} u_{m 0}\right|^{2}\right)\right)+\left|A u_{m 0}\right|^{2}$.

Let $\left[0, T^{*}\right)$ be the maximal interval where the solution exists. Set $Z(t):=M\left(\left|A^{1 / 2} u_{m}(t)\right|^{2}\right)$ and

$$
T:=\sup \left\{\tau \in\left[0, T^{*}\right)|| \frac{Z^{\prime}(t)}{Z(t)} \mid \leq \frac{1}{2}, Z(t)>0, \forall t \in[0, \tau)\right\} .
$$

With simple computations it follows that

$$
\begin{aligned}
P^{\prime}(t) & =-\frac{1}{Z(t)}\left(2\left(g\left(u_{m}^{\prime}(t)\right), u_{m}^{\prime}(t)\right)+\frac{Z^{\prime}(t)}{Z(t)}\left|u_{m}^{\prime}(t)\right|^{2}\right) \\
& \leq 0
\end{aligned}
$$

$Q^{\prime}(t)$

$$
\begin{aligned}
& =-\frac{1}{Z(t)}\left(2\left(g\left(u_{m}^{\prime}(t)\right), A u_{m}^{\prime}(t)\right)+\frac{Z^{\prime}(t)}{Z(t)}\left|A^{1 / 2} u_{m}^{\prime}(t)\right|^{2}\right) \\
& \leq 0
\end{aligned}
$$

$\left(G^{2}\right)^{\prime}(t)$

$$
\leq-G(t)\left\{2\left(\frac{Z^{\prime}(t)}{Z(t)}-\left|g\left(u_{m}^{\prime}(t)\right)\right|\right) G(t)-2\left|A u_{m}(t)\right|\right\},
$$

for all $t \in[0, T)$.

Next, we show that $T=T^{*}$. Let us assume by contradiction that $T<T^{*}$. Since $\left|Z^{\prime}(t)\right| \leq(1 / 2) Z(t)$ in $[0, T)$, we have that

$$
0<Z(0) e^{-T / 2} \leq Z(T) \leq Z(0) e^{T / 2} .
$$


Since $Z(t)$ and $Z^{\prime}(t)$ are continuous functions, by the maximality of $T$ we have that necessarily

$$
\left|\frac{Z^{\prime}(t)}{Z(t)}\right|=\frac{1}{2}
$$

From (88) and (89) it follows that $P$ and $Q$ are nonincreasing functions; hence

$$
\begin{gathered}
\left|A^{1 / 2} u_{m}(t)\right|^{2} \leq P(t) \leq P(0), \\
\left|A u_{m}(t)\right|^{2} \leq Q(t) \leq Q(0) .
\end{gathered}
$$

Moreover by Lemma 3.1 in [28] we have that

$$
G(t) \leq \max \left\{G(0), \frac{Z(0)}{Z^{\prime}(0)-g\left(u_{m 1}\right) Z(0)} \sqrt{Q(0)}\right\},
$$$$
\forall t \in[0, T] .
$$

By (91)-(31), and the smallness assumption (23), we have that

$$
\begin{aligned}
\left|\frac{Z^{\prime}(T)}{Z(T)}\right|= & \left|\frac{2 M^{\prime}\left(\left|A^{1 / 2} u_{m}(t)\right|^{2}\right)\left(u_{m}^{\prime}(T), A u_{m}(T)\right)}{Z(T)}\right| \\
\leq & 2 \max _{0 \leq r \leq P(0)}\left|M^{\prime}(r)\right| \frac{\left|u_{m}^{\prime}(T)\right|}{Z(T)}\left|A u_{m}(T)\right| \\
\leq & 2 \max _{0 \leq r \leq P(0)}\left|M^{\prime}(r)\right| \\
& \times \max \left\{G(0), \frac{Z(0)}{Z^{\prime}(0)-g\left(u_{m 1}\right) Z(0)} \sqrt{Q(0)}\right\} \\
& \times \sqrt{Q(0)} \\
< & \frac{1}{2} .
\end{aligned}
$$

This contradicts (29). Therefore it follows that $u_{m}(t)$ can be extended to $[0, T)$ for any $T \in(0, \infty)$.

Furthermore, putting $v=A u_{m}^{\prime}$ in (13), we get

$$
\frac{\left(K u_{m}^{\prime \prime}, A u_{m}^{\prime}\right)}{M\left(\left|A^{1 / 2} u_{m}\right|^{2}\right)}+\left(A u_{m}, A u_{m}^{\prime}\right)+\frac{\left(g\left(u_{m}^{\prime}\right), A u_{m}^{\prime}\right)}{M\left(\left|A^{1 / 2} u_{m}\right|^{2}\right)}=0
$$

From this we obtain

$$
\begin{aligned}
\frac{1}{2} \frac{d}{d t}\left(\frac{\left(K u_{m}^{\prime}, A u_{m}^{\prime}\right)}{M\left(\left|A^{1 / 2} u_{m}\right|^{2}\right)}+\left|A u_{m}\right|^{2}\right)+\frac{\left(g\left(u_{m}^{\prime}\right), A u_{m}^{\prime}\right)}{M\left(\left|A^{1 / 2} u_{m}\right|^{2}\right)} \\
=-\frac{\left(K u_{m}^{\prime}, A u_{m}^{\prime}\right) M^{\prime}\left(\left|A^{1 / 2} u_{m}\right|^{2}\right)\left(A^{1 / 2} u_{m}^{\prime}, A^{1 / 2} u_{m}\right)}{\left\{M\left(\left|A^{1 / 2} u_{m}\right|^{2}\right)\right\}^{2}} .
\end{aligned}
$$

Integrating $(34)$ over $(0, t)$ and taking into account assumptions $(\mathrm{M})$ and $(\mathrm{G})$, and applying Gronwall's inequality, we obtain

$$
A u_{m} \text { bounded in } L^{\infty}(0, T ; H)
$$

From (6) and (22), it follows that

$$
g\left(u_{m}^{\prime}\right) \text { bounded in } L^{(q+1) / q}([0, T] \times \Omega) .
$$

A Priori Estimate II. Taking $v=u_{m}^{\prime \prime}(t)$ in (13) and choosing $t=0$, we obtain

$$
\begin{aligned}
& \left|K^{1 / 2} u_{m}^{\prime \prime}(0)\right|^{2}+\left(M\left(\left|A^{1 / 2} u_{0 m}\right|^{2}\right) A u_{0 m}+g\left(u_{1 m}\right), u_{m}^{\prime \prime}(0)\right) \\
& \quad=0 .
\end{aligned}
$$

Thus we have

$$
\begin{aligned}
\left|K^{1 / 2} u_{m}^{\prime \prime}(0)\right|^{2} \leq & \left(\left|g\left(u_{1 m}\right)\right|+\left|M\left(\left|A^{1 / 2} u_{0 m}\right|^{2}\right) A u_{0 m}\right|\right)\left|u_{m}^{\prime \prime}(0)\right| \\
\leq & \left(\left|g\left(u_{1 m}\right)\right|+\left|M\left(\left|A^{1 / 2} u_{0}\right|^{2}\right) A u_{0}\right|\right) \\
& \times\left|K^{1 / 2} u_{m}^{\prime \prime}(0)\right| .
\end{aligned}
$$

Thanks to the assumption (6), we deduce from (15) that

$$
\left(g\left(u_{1 m}\right)\right) \text { is bounded in } L^{2}(\Omega) \text {. }
$$

Therefore we conclude that the right-hand side is bounded; that is,

$$
K^{1 / 2} u_{m}^{\prime \prime}(0) \text { bounded in } H
$$

A Priori Estimate III. For $t<T$, we apply (13) at points $t$ and $t+$ $\zeta$ such that $0<\zeta<T-t$. By taking the difference $v=u_{m}^{\prime}(t+$ $\zeta)-u_{m}^{\prime}(t)$ in (13) and the assumption $(\mathrm{G})$, we obtain

$$
\begin{aligned}
0 \geq & \left(K u_{m}^{\prime \prime}(t+\zeta)-K u_{m}^{\prime \prime}(t), u_{m}^{\prime}(t+\zeta)-u_{m}^{\prime}(t)\right) \\
+ & \left(M\left(\left|A^{1 / 2} u_{m}(t+\zeta)\right|^{2}\right) A u_{m}(t+\zeta)\right. \\
& \left.\quad M\left(\left|A^{1 / 2} u_{m}(t)\right|^{2}\right) A u_{m}(t), u_{m}^{\prime}(t+\zeta)-u_{m}^{\prime}(t)\right) .
\end{aligned}
$$

Thus we have

$$
\begin{aligned}
0 \geq \frac{1}{2} & \frac{d}{d t}\left[\left|K^{1 / 2}\left(u_{m}^{\prime}(t+\zeta)-u_{m}^{\prime}(t)\right)\right|^{2}\right] \\
& +M\left(\left|A^{1 / 2} u_{m}(t+\zeta)\right|^{2}\right) \\
& \times\left(A u_{m}(t+\zeta)-A u_{m}(t), u_{m}^{\prime}(t+\zeta)-u_{m}^{\prime}(t)\right) \\
& +\left[M\left(\left|A^{1 / 2} u_{m}(t+\zeta)\right|^{2}\right)-M\left(\left|A^{1 / 2} u_{m}(t)\right|^{2}\right)\right] \\
& \times\left(A u_{m}(t), u_{m}^{\prime}(t+\zeta)-u_{m}^{\prime}(t)\right) .
\end{aligned}
$$

Set

$$
\boldsymbol{\Phi}_{\zeta m}(t)=\left|K^{1 / 2}\left(u_{m}^{\prime}(t+\zeta)-u_{m}^{\prime}(t)\right)\right|^{2}
$$


By using (42), Young's inequality, the assumption (M), and the fact that $K$ is positive self-adjoint operator, we see that $\Phi_{\zeta m}^{\prime}(t) \leq c \Phi_{\zeta m}(t)$. Therefore we deduce

$$
\boldsymbol{\Phi}_{\zeta m}(t) \leq \Phi_{\zeta m}(0) \exp (c T) \quad \forall t \in[0, T]
$$

Dividing the two sides of (44) by $\zeta^{2}$, letting $\zeta \rightarrow 0$, and using (43), we deduce

$$
c\left|u_{m}^{\prime \prime}\right|^{2} \leq\left|K^{1 / 2} u_{m}^{\prime \prime}(0)\right|^{2}
$$

From (40), it follows that $\left|u_{m}^{\prime \prime}\right|^{2} \leq C$.

Since $u_{m} \in C^{2}[0, T]$, the previous inequality is verified for all $t \in[0, T]$. Therefore we conclude that

$$
u_{m}^{\prime \prime} \text { bounded in } L^{\infty}(0, T ; H) \text {. }
$$

Moreover, using (19) and (46), it follows that

$$
\begin{aligned}
& u_{m}^{\prime} \text { bounded in } L^{2}(0, T ; H), \\
& u_{m}^{\prime \prime} \text { bounded in } L^{2}(0, T ; H) .
\end{aligned}
$$

Applying a compactness theorem given in [33], we obtain

$$
u_{m}^{\prime} \text { precompact in } L^{2}(0, T ; H) \text {. }
$$

Passage to the Limit. Applying the Dunford-Pettis theorem, we conclude from (19), (21), (36), and (46)-(48), replacing the sequence $u_{m}$ with a subsequence if needed, that

$$
\begin{gathered}
u_{m} \longrightarrow u \text { weak-star in } L^{\infty}(0, T ; V), \\
u_{m}^{\prime} \longrightarrow u^{\prime} \text { weak-star in } L^{\infty}(0, T ; H), \\
u_{m}^{\prime \prime} \longrightarrow u^{\prime \prime} \text { weak-star in } L^{\infty}(0, T ; H), \\
u_{m}^{\prime} \longrightarrow u^{\prime} \text { a.e in } \Omega \times[0, T], \\
g\left(u_{m}^{\prime}\right) \longrightarrow \psi \text { weak-starin } L^{(q+1) / q}(0, T ; H), \\
M\left(\left|A^{1 / 2} u_{m}\right|^{2}\right) A u_{m} \longrightarrow \chi \text { weak-star in } L^{\infty}(0, T ; H)
\end{gathered}
$$

for suitable functions $u \in L^{\infty}(0, T ; V), \chi \in L^{\infty}(0, T ; H)$, and $\psi \in L^{(q+1) / q}(\Omega \times[0, T])$.

Now we are going to show that $u$ is a solution of the problem (11)-(12). Indeed, from (49) to (51), we have

$$
\begin{aligned}
& \int_{\Omega} u_{m}(0) e^{j} d x \rightarrow \int_{\Omega} u(0) e^{j} d x, \\
& \int_{\Omega} u_{m}^{\prime}(0) e^{j} d x \rightarrow \int_{\Omega} u^{\prime}(0) e^{j} d x
\end{aligned}
$$

for each fixed $j \geq 1$. So we conclude that, for any $j \geq 1$,

$$
\begin{array}{r}
\int_{\Omega}\left(u_{m}(0)-u_{0}\right) e^{j} d x=\int_{\Omega}\left(u^{\prime}(0)-u_{1}\right) e^{j} d x=0 \\
\text { as } m \longrightarrow \infty,
\end{array}
$$

which shows that (12) holds.
We will prove that, in fact, $\chi=M\left(\left|A^{1 / 2} u\right|^{2}\right) A u$; that is,

$$
\begin{array}{r}
M\left(\left|A^{1 / 2} u_{m}\right|^{2}\right) A u_{m} \longrightarrow M\left(\left|A^{1 / 2} u\right|^{2}\right) A u \\
\text { weak-star in } L^{\infty}(0, \infty ; H) .
\end{array}
$$

For $v \in L^{2}(0, T ; H)$, we have

$$
\begin{aligned}
& \int_{0}^{T}\left(\chi-M\left(\left|A^{1 / 2} u\right|^{2}\right) A u, v\right) d t \\
& =\int_{0}^{T}\left(\chi-M\left(\left|A^{1 / 2} u_{m}\right|^{2}\right) A u_{m}, v\right) d t \\
& \quad+\int_{0}^{T} M\left(\left|A^{1 / 2} u\right|^{2}\right)\left(A u_{m}-A u, v\right) d t \\
& \quad+\int_{0}^{T}\left(M\left(\left|A^{1 / 2} u_{m}\right|^{2}\right)-M\left(\left|A^{1 / 2} u\right|^{2}\right)\right) \\
& \quad \times\left(A u_{m}, v\right) d t
\end{aligned}
$$

We deduce from (49) and (54) that the first and second terms in (58) tend to zero as $m \rightarrow \infty$. For the last term, using the fact that $M$ is $C^{1}$ and (21), we can derive (with some positive constants $c_{1}, c_{2}$ )

$$
\begin{aligned}
& \int_{0}^{T}\left(M\left(\left|A^{1 / 2} u_{m}\right|^{2}\right)-M\left(\left|A^{1 / 2} u\right|^{2}\right)\right)\left(A u_{m}, v\right) d t \\
& \quad \leq c_{1} \int_{0}^{T}\left|A\left(u_{m}+u\right), u_{m}-u\right| d t \\
& \quad \leq c_{2}\left(\int_{0}^{T}\left|u_{m}-u\right|^{2} d t\right)^{1 / 2} .
\end{aligned}
$$

Since $u_{m}$ is bounded in $L^{\infty}(0, T ; V)$ and the injection of $V$ in $H$ is compact, we have

$$
u_{m} \longrightarrow u \text { strongly in } L^{2}(0, T ; H)
$$

From (58) to (60), we deduce (57). It follows from (49), (51), and (57) that, for each fixed $v \in L^{q+1}(0, T ; V)$,

$$
\begin{aligned}
& \int_{0}^{T}\left(K u_{m}^{\prime \prime}+M\left(\left|A^{1 / 2} u_{m}\right|^{2}\right) A u_{m}, v\right) d t \\
& \longrightarrow \int_{0}^{T}\left(K u^{\prime \prime}+M\left(\left|A^{1 / 2} u\right|^{2}\right) A u, v\right) d t
\end{aligned}
$$

as $m \rightarrow+\infty$

For the nonlinear term, $g\left(u^{\prime}\right)$, it remains to show that, for any fixed $v \in L^{q+1}(0, T ; V)$,

$$
\int_{0}^{T} \int_{\Omega} v g\left(u_{m}^{\prime}\right) d x d t \longrightarrow \int_{0}^{T} \int_{\Omega} v g\left(u^{\prime}\right) d x d t
$$

as $m \rightarrow \infty$

At this moment we use the following lemma due to Jung and Choi (see [26, page 12]). 
Lemma 2. Suppose that $\Omega \times[0, T]$ is a bounded open domain of $\mathbb{R}^{n} \times \mathbb{R} ; g_{m}$ and $g$ are in $L^{q}(\Omega \times[0, T]), 1<q<\infty$, such that $g_{m} \rightarrow g$ a.e., in $\Omega \times[0, T]$. Then $g_{m} \rightarrow g$ weakly in $L^{q}(\Omega \times$ $[0, T])$.

From (53), $g\left(u_{m}^{\prime}\right) \rightarrow g\left(u^{\prime}\right)$ a.e. in $\Omega \times[0, T]$. By (36), we can use the above lemma and so we have $\psi=g\left(u^{\prime}\right)$; that is,

$$
g\left(u_{m}\right) \longrightarrow g(u) \text { weak in } L^{(q+1) / q}(\Omega \times(0, T)),
$$

which implies (62). Therefore we obtain

$$
\begin{array}{r}
\int_{0}^{T}\left(K u^{\prime \prime}+M\left(\left|A^{1 / 2} u\right|^{2}\right) A u+g\left(u^{\prime}\right), v\right) d t=0, \\
\forall v \in L^{q+1}(0, T ; V) .
\end{array}
$$

The uniqueness is obtained by a standard method, so we omit the proof here.

\section{Energy Estimates}

In this section we study the energy estimate under suitable growth conditions on $g$.

Let us assume that there exist a number $p \geq 1$ and positive constants $c_{1}, i=1,2$, such that

$$
\begin{aligned}
& c_{1} \min \left\{\left|K^{1 / 2} x\right|,\left|K^{1 / 2} x\right|^{p}\right\} \\
& \quad \leq|g(x)| \leq c_{2} \max \left\{\left|K^{1 / 2} x\right|,\left|K^{1 / 2} x\right|^{1 / p}\right\}
\end{aligned}
$$

for all $x \in \mathbb{R}$.

Theorem 3. Assume that (65) holds. Then one obtains the following energy decay:

$$
E(t) \leq \begin{cases}c_{0} E(0) e^{-w t} \forall t \geq 0, & \text { if } p=1, \\ \widetilde{\mathcal{c}}_{0}(1+t)^{-2 /(p-1)} \forall t \geq 0, & \text { if } p>1,\end{cases}
$$

where $c_{0}, w$, and $\widetilde{c}_{0}$ are some positive constants.

Proof. Let $T>0$ be arbitrary and fixed and let $u \in L^{\infty}(0$, $T ; V) \cap W^{2, \infty}(0, T ; H)$ be a solution of (11) and (12). Multiplying (11) by $u^{\prime}$ and integrating by parts in $\Omega \times(s, T)(0 \leq s<T)$, we obtain that

$$
E(T)-E(s)=-\int_{s}^{T}\left(g\left(u^{\prime}(t)\right), u^{\prime}(t)\right) d t .
$$

By $\left(g\left(u^{\prime}(t)\right), u^{\prime}(t)\right) \geq 0$ and being the primitive of an integrable function, it follows that the energy $E$ is nonincreasing, locally absolutely continuous and

$$
E^{\prime}(t)=-\left(g\left(u^{\prime}(t)\right), u^{\prime}(t)\right) \quad \text { a.e. in }[0, \infty) .
$$

Here and in what follows we will denote by $c$ diverse positive constants. We are going to show that the energy of this solution satisfies

$$
\int_{s}^{T} E(t)^{(p+1) / 2} \leq c E(s) \quad \forall 0 \leq s \leq T<\infty .
$$

Once (69) is satisfied, the integral inequalities given in Komornik [31] and Haraux [32] will establish (66).

Now, multiplying (11) by $E(t)^{(p-1) / 2} u$ and integrating by parts, we have

$$
\begin{aligned}
0= & \int_{s}^{T} E(t)^{(p-1) / 2}\left(K u^{\prime \prime}+M\left(\left|A^{1 / 2} u\right|^{2}\right) A u+g\left(u^{\prime}\right), u\right) d t \\
= & {\left[E(t)^{(p-1) / 2}\left(K u^{\prime}, u\right)\right]_{s}^{T} } \\
& -\frac{p-1}{2} \int_{s}^{T} E(t)^{(p-3) / 2} E^{\prime}(t)\left(K u^{\prime}, u\right) d t \\
& -\int_{s}^{T} E(t)^{(p-1) / 2}\left|K^{1 / 2} u^{\prime}\right|^{2} d t \\
& +\int_{s}^{T} E(t)^{(p-1) / 2}\left(M\left(\left|A^{1 / 2} u\right|^{2}\right)\left|A^{1 / 2} u\right|^{2}, u\right) d t \\
& +\int_{s}^{T} E(t)^{(p-1) / 2}\left(g\left(u^{\prime}\right), u\right) d t .
\end{aligned}
$$

Note that by the assumption (M) and (21), we can choose some positive number

$$
\alpha=\max _{s \in\left[0,\left|A^{1 / 2} u\right|^{2}\right]}\{M(s)\}<\infty
$$

so that $2 E(t) \leq\left|K^{1 / 2} u^{\prime}\right|^{2}+\alpha\left|A^{1 / 2} u\right|^{2}$. Thus we deduce that

$$
\begin{aligned}
\frac{2 \beta}{\alpha} \int_{s}^{T} E(t)^{(p+1) / 2} d t & \\
\leq & -\left[E(t)^{(p-1) / 2}\left(K u^{\prime}, u\right)\right]_{s}^{T} \\
& +\frac{p-1}{2} \int_{s}^{T} E(t)^{(p-3) / 2} E^{\prime}(t)\left(K u^{\prime}, u\right) d t \\
& +\int_{s}^{T} E(t)^{(p-1) / 2}\left(\left(1+\alpha^{-1}\right)\left|K^{1 / 2} u^{\prime}\right|^{2}-\left(g\left(u^{\prime}\right), u\right)\right) d t \\
\equiv & I_{1}+I_{2}+I_{3} .
\end{aligned}
$$

Using the continuity of the imbedding $V \subset H$, the CauchySchwarz and the Young inequalities, we obtain

$$
\left|\left(K u^{\prime}, u\right)\right| \leq c\left|K u^{\prime}\right|\|u\| \leq c E(t) .
$$

Hence, since $E(t)$ is nonincreasing, we obtain

$$
\begin{aligned}
I_{1} & \leq c E^{(p-1) / 2}(0) E(s), \\
I_{2} & \leq \frac{(p-1)}{2} \int_{s}^{T} c E(t)^{(p-1) / 2} E^{\prime}(t) d t \\
& \leq c E^{(p-1) / 2}(0) E(s) .
\end{aligned}
$$

In order to estimate the last term $I_{3}$ of (72), we set

$$
\begin{aligned}
& \Omega_{1}=\left\{x \in \Omega:\left|K^{1 / 2} u^{\prime}(t, x)\right| \leq 1\right\}, \\
& \Omega_{2}=\left\{x \in \Omega:\left|K^{1 / 2} u^{\prime}(t, x)\right|>1\right\} .
\end{aligned}
$$


Then we have

$$
\begin{aligned}
\int_{\Omega}\left|K^{1 / 2} u^{\prime}(t, x)\right|^{2} d x= & \int_{\Omega_{1}}\left|K^{1 / 2} u^{\prime}(t, x)\right|^{2} d x \\
& +\int_{\Omega_{2}}\left|K^{1 / 2} u^{\prime}(t, x)\right|^{2} d x .
\end{aligned}
$$

The Hölder inequality yields

$$
\begin{aligned}
\int_{\Omega}\left|K^{1 / 2} u^{\prime}(t, x)\right|^{2} d x \leq & c\left(\int_{\Omega_{1}}\left|K^{1 / 2} u^{\prime}(t, x)\right|^{p+1} d x\right)^{2 /(p+1)} \\
& +\int_{\Omega_{2}}\left|K^{1 / 2} u^{\prime}(t, x)\right|^{2} d x \\
& \equiv J_{1}+J_{2} .
\end{aligned}
$$

Using (65) and (68), we deduce that

$$
\begin{aligned}
& J_{1} \leq c\left(\int_{\Omega_{1}} u^{\prime} g\left(u^{\prime}\right) d x\right)^{2 /(p+1)} \leq c\left|E^{\prime}(t)\right|^{2 /(p+1)}, \\
& J_{2} \leq c \int_{\Omega_{2}}\left|u^{\prime} g\left(u^{\prime}\right)\right| d x \leq c\left(-E^{\prime}(t)\right) .
\end{aligned}
$$

Combining these two inequalities with (77), we obtain

$$
\int_{\Omega}\left|K^{1 / 2} u^{\prime}(t, x)\right|^{2} d x \leq c\left(-E^{\prime}(t)\right)^{2 /(p+1)}+c\left(-E^{\prime}(t)\right) .
$$

Applying Young's inequality, it follows that, for any $\epsilon>0$,

$$
\begin{aligned}
& \int_{s}^{T} E(t)^{(p-1) / 2}\left|K^{1 / 2} u^{\prime}\right|^{2} d t \\
& \leq \epsilon c \int_{s}^{T} E(t)^{(p+1) / 2} d t \\
& \quad+c\left(\epsilon^{(1-p) / 2}+E^{(p-1) / 2}(0)\right) E(s) .
\end{aligned}
$$

It remains to estimate the second term of $I_{3}$. Using (88) we have

$$
\begin{aligned}
& \left|\int_{\Omega_{1}} u g\left(u^{\prime}\right) d x\right| \\
& \quad \leq c\|u\|_{L^{(p+1) / p}\left(\Omega_{1}\right)}\left\|g\left(u^{\prime}\right)\right\|_{L^{p+1}\left(\Omega_{1}\right)} \\
& \quad \leq c\|u\|_{L^{(p+1) / p}\left(\Omega_{1}\right)}\left(\int_{\Omega_{1}} u^{\prime} g\left(u^{\prime}\right) d x\right)^{1 /(p+1)} \\
& \quad \leq c E(t)^{1 / 2}\left(-E^{\prime}(t)\right)^{1 /(p+1)} .
\end{aligned}
$$

Similarly, using (6), we obtain

$$
\begin{aligned}
\left|\int_{\Omega_{2}} u g\left(u^{\prime}\right) d x\right| & \leq c\|u\|_{L^{2}\left(\Omega_{2}\right)}\left\|g\left(u^{\prime}\right)\right\|_{L^{2}\left(\Omega_{2}\right)} \\
& \leq c\|u\|_{L^{2}\left(\Omega_{2}\right)}\left\|u^{\prime} g\left(u^{\prime}\right)\right\|_{L^{1}\left(\Omega_{2}\right)}^{1 / 2} \\
& \leq c E(t)^{1 / 2}\left(-E^{\prime}(t)\right)^{1 / 2} .
\end{aligned}
$$

From (81) and (82), we deduce

$$
\begin{aligned}
\left|\int_{\Omega} u g\left(u^{\prime}\right) d x\right| \leq & c E(t)^{1 / 2}\left(-E^{\prime}(t)\right)^{1 /(p+1)} \\
& +c E(t)^{1 / 2}\left(-E^{\prime}(t)\right)^{1 / 2}
\end{aligned}
$$

Using Young's inequality and

$$
E(t)^{p / 2}\left(-E^{\prime}(t)\right)^{1 / 2}=E(t)^{(p+1) / 4}\left(E(t)^{(p-1) / 4}\left(-E^{\prime}(t)\right)^{1 / 2}\right),
$$

it follows from (82) that, for any $\epsilon>0$,

$$
\begin{aligned}
& -\int_{s}^{T} E(t)^{(p-1) / 2}\left(g\left(u^{\prime}\right), u\right) d t \\
& =-\int_{s}^{T} E(t)^{(p-1) / 2} \int_{\Omega} u g\left(u^{\prime}\right) d x d t \\
& \leq c \int_{s}^{T} E(t)^{p / 2}\left(-E^{\prime}(t)\right)^{1 /(p+1)} d t \\
& +c \int_{s}^{T} E(t)^{p / 2}\left(-E^{\prime}(t)\right)^{1 / 2} d t \\
& \leq 2 \epsilon c \int_{s}^{T} E(t)^{(p+1) / 2} d t \\
& \quad+c\left(\epsilon^{-p}+\epsilon^{-1} E(0)^{(p-1) / 2}\right) \\
& \quad \times \int_{s}^{T}\left(-E^{\prime}(t)\right) d t \\
& \leq 2 \epsilon c \int_{s}^{T} E(t)^{(p+1) / 2} d t \\
& +c\left(\epsilon^{-p}+\epsilon^{-1} E(0)^{(p-1) / 2}\right) E(s) .
\end{aligned}
$$

Combining (80) with (85) and setting $\widetilde{\alpha}=1+\alpha^{-1}$, we obtain

$$
\begin{aligned}
I_{3} \leq & \int_{s}^{T} E(t)^{(p-1) / 2} 2\left|K^{1 / 2} u^{\prime}\right|^{2} d t \\
& +\int_{s}^{T} E(t)^{(p-1) / 2} \int_{\Omega} u g\left(u^{\prime}\right) d x d t \\
\leq & (\widetilde{\alpha}+2) \epsilon c \int_{s}^{T} E(t)^{(p+1) / 2} d t \\
& +c\left(\widetilde{\alpha} \epsilon^{(1-p) / 2}+\epsilon^{-p}\right. \\
& \left.\quad+\left(\widetilde{\alpha}+\epsilon^{-1}\right) E(0)^{(p-1) / 2}\right) E(s) .
\end{aligned}
$$

Therefore we conclude that

$$
\begin{aligned}
&\left(\frac{2 \beta}{\alpha}-\right.(\widetilde{\alpha}+2) \epsilon c) \int_{s}^{T} E^{(p+1) / 2} \\
& \leq c\left(\widetilde{\alpha} \epsilon^{(1-p) / 2}+\epsilon^{-p}\right. \\
&\left.\quad+\left(\widetilde{\alpha}+\epsilon^{-1}\right) E(0)^{(p-1) / 2}\right) E(s) .
\end{aligned}
$$

Now we choose $\epsilon$ as $\epsilon \in(0,2 \beta /(3 \alpha+1) c)$; then (69) follows. 
TABLE 1: Simulation parameters which are satisfied by theoretical conditions.

\begin{tabular}{lccc}
\hline Symbols & Definition & Values & Reference \\
\hline$A(x)$ & Cross-sectional area & $0.7853\left(10^{-4} \sin \left(2^{10} \pi x\right)+1\right) \mathrm{cm}^{2}$ & {$[34]$} \\
$\rho$ & Mass density of the unit length & $7.850 \mathrm{~g} / \mathrm{cm}^{2}$ & {$[34]$} \\
\hline
\end{tabular}

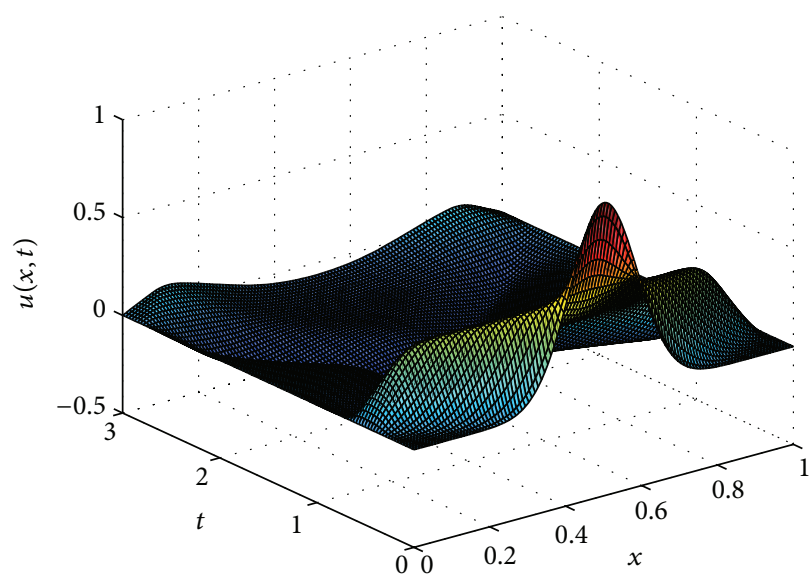

(a) Temporal and spatial solution shapes in case of $\kappa=10$

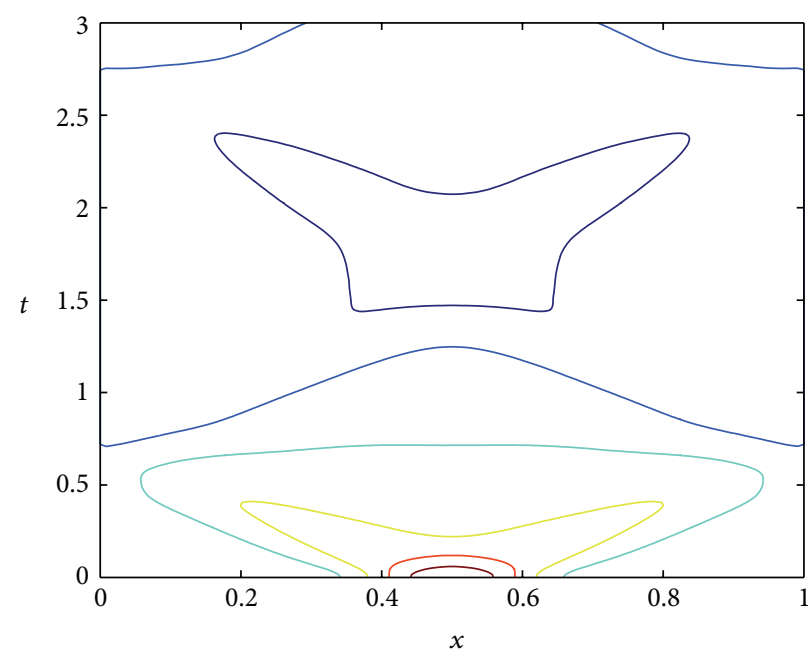

(c) Temporal and spatial solution contour line in case of $\kappa=10$

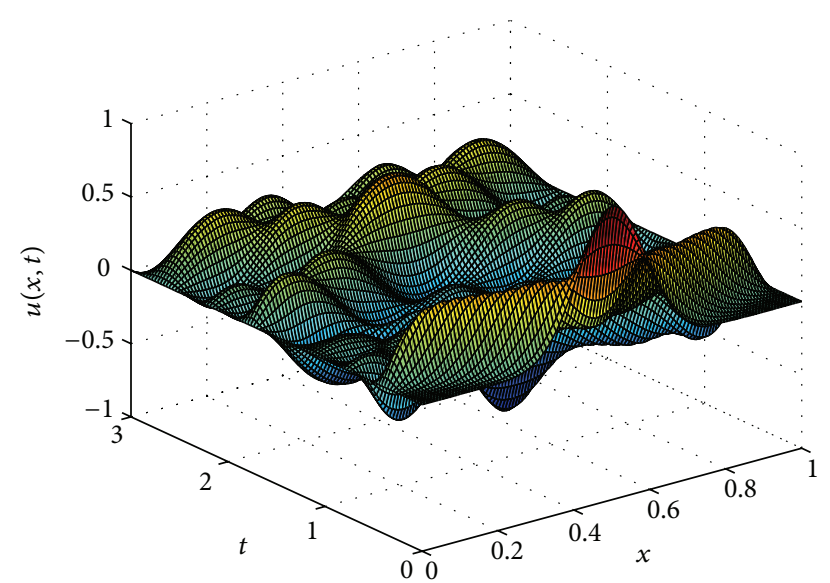

(b) Temporal and spatial solution shapes in case of $\kappa=10^{-0.3}$

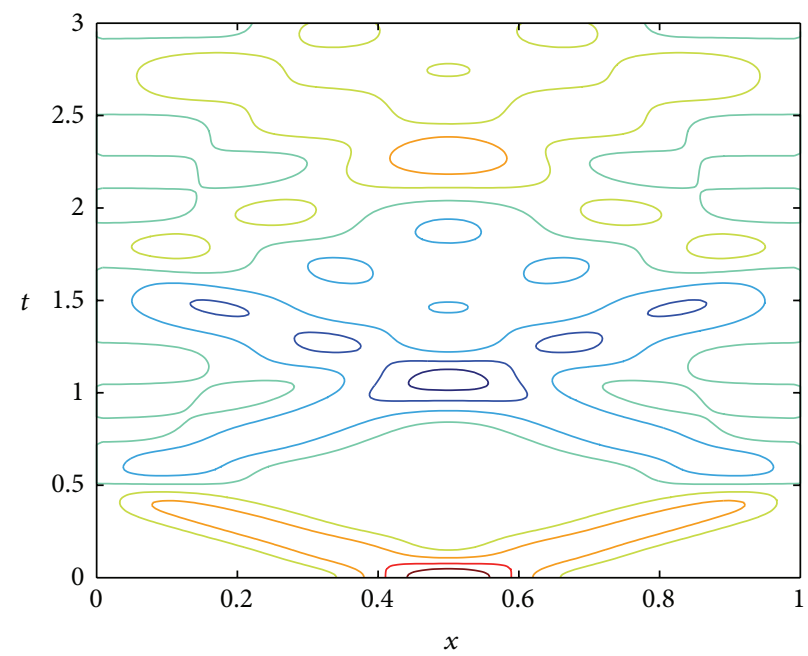

(d) Temporal and spatial solution contour line in case of $\kappa=10^{-0.3}$

FIGURE 1: Solution shapes and contour lines with respect to $\kappa=10$ and $\kappa=10^{-0.3}$.

\section{Numerical Result}

In this section, we consider a Kirchhoff-type equation with heterogeneous string as an application:

$$
\begin{gathered}
(A(x) \rho) u^{\prime \prime}(x, t)-\left(1+\int_{0}^{1}|\nabla u(x, t)|^{2} d x\right) \Delta u(x, t) \\
+\kappa\left|u^{\prime}(x, t)\right|^{2} u^{\prime}(x, t)=0, \\
\operatorname{in}(x, t) \in(0,1) \times(0,3), \\
u(0, t)=u(1, t)=0 \text { on }(0,3),
\end{gathered}
$$

$$
\begin{gathered}
u_{0}=u(x, 0)=\exp \left(-64\left(x-\frac{1}{2}\right)^{2}\right) \text { in }(0,1), \\
u_{1}=u_{t}(x, 0)=0 \quad \text { in }(0,1)
\end{gathered}
$$

where $\kappa$ is a positive constant and $A(x), \rho$ are given in Table 1.

Then, the operators $K=A(x) \rho I(I: H \rightarrow H$; identity operator), $A=-\Delta$, and the functions $M(s)=s+$ 1 and $g(x)=\kappa|x|^{2} x$ so that we can easily check that the hypotheses $(\mathrm{M}),(\mathrm{G}),(\mathrm{H})$, and $(\mathrm{S})$ in Preliminaries are satisfied. The smallness condition satisfies $\left(\left\|\nabla u_{0}\right\|^{2}+1\right)\left\|\Delta u_{0}\right\|^{2} \approx$ $0.213 \leq 1 / 4$. Therefore, by Theorem 1 , we can deduce the following results. 


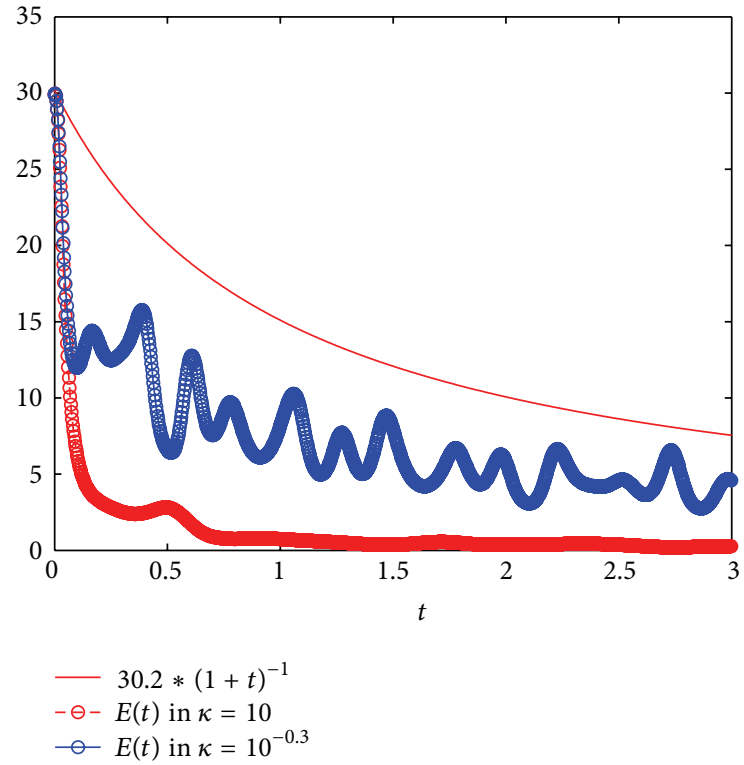

Figure 2: Algebraic decay rates of the energy in case of $\kappa=10$ and $\kappa=10^{-0.3}$.

Theorem 4. For any $T>0$, there is a unique solution $u \in$ $L^{\infty}\left(0, T ; H^{2}(0,1)\right) \cap W^{1, \infty}\left(0, T ; H_{0}^{1}(0,1)\right) \cap W^{2, \infty}\left(0, T ; L^{2}(0\right.$, 1)) to the system (88)-(92).

The energy for the system (88)-(92) is given by

$$
\begin{gathered}
E(t)=\frac{1}{2}\left[\int_{0}^{1} \mid \sqrt{\left.A(x) \rho u^{\prime}(x, t)\right|^{2} d x+\int_{0}^{1}|\nabla u(x, t)|^{2} d x}\right. \\
\left.+\frac{1}{2}\left(\int_{0}^{1}|\nabla u(x, t)|^{2} d x\right)^{2}\right] .
\end{gathered}
$$

Next, in order to get the energy decay of (88)-(92), we need the value of the parameter $p$ in (65). We can easily check that $p=3$ when $g(x)=\kappa|x|^{2} x$.

Therefore, by Theorem 3, we get the energy decay rates for the energy $E(t)$ as follows.

Theorem 5. We obtain the following energy decay:

$$
E(t) \leq c_{1}(1+t)^{-1} \quad \forall t \geq 0,
$$

where $c_{1}$ is a positive constant.

For the numerical simulation, we use the finite difference methods (FDM) which are the implicit multistep methods in time and second-order central difference methods for the space derivative in space in numerical algorithms (see $[8,9$, 11]).

Figures 1(a)-1(d) show displacements of solutions to the system (88)-(92) with $\kappa=10$ and $\kappa=10^{-0.3}$, respectively.

In case of $\kappa=10$ and $\kappa=10^{-0.3}$, we deduce the algebraic decay rate for the energy as shown in Figure 2, respectively. The blue line and red dotted circled line (or blue circled line) show $c_{1}(t+1)^{1}$ and $E(t)$ per the two values, respectively, where the parameter value $c_{1}=30.2$ in (94). This result shows that the energy decay rates for solutions are algebraic in case that the system (88)-(92) with the nonlinear damping term $\kappa\left|u_{t}\right|^{2} u_{t}$.

\section{Conflict of Interests}

The authors declare that there is no conflict of interests regarding the publication of this paper.

\section{Acknowledgments}

The first author's research was supported by Basic Research Program through the National Research Foundation of Korea (NRF) funded by the Ministry of Education, Science and Technology (Grant no. NRF-2013R1A1A2010704). The corresponding author's research was supported by Basic Research Program through the National Research Foundation of Korea (NRF) funded by the Ministry of Education, Science and Technology (Grant no. NRF-2012R1A1B3000599).

\section{References}

[1] G. Kirchhoff, Vorlesungen Über Mechanik, Teubner, Leipzig, Germany, 1883.

[2] Q. C. Nguyen and K. -S. Hong, "Transverse vibration control of axially moving membranes by regulation of axial velocity," IEEE Transactions on Control Systems Technology, vol. 20, no. 4, pp. 1124-1131, 2012.

[3] G. P. Menzala, A. F. Pazoto, and E. Zuazua, "Stabilization of Berger-Timoshenko's equation as limit of the uniform stabilization of the von Kármán system of beams and plates," Mathematical Modelling and Numerical Analysis, vol. 36, no. 4, pp. 657-691, 2002.

[4] J. G. Eisley, "Nonlinear vibration of beams and rectangular plates," Zeitschrift für Angewandte Mathematik und Physik, vol. 15, no. 2, pp. 167-175, 1964.

[5] S. Woinowsky-Krieger, "The effect of an axial force on the vibration of hinged bars," Journal of Applied Mechanics, vol. 17, pp. 35-36, 1950.

[6] L. Chen, C. W. Lim, and H. Ding, "Energetics and conserved quantity of an axially moving string undergoing three-dimensional nonlinear vibration," Acta Mechanica Sinica, vol. 24, no. 2 , pp. 215-221, 2008.

[7] K.-S. Hong, "Asymptotic behavior analysis of a coupled timevarying system: application to adaptive systems," IEEE Transactions on Automatic Control, vol. 42, no. 12, pp. 1693-1697, 1997.

[8] D. Kim, S. Kim, and I. H. Jung, "Stabilization for the Kirchhoff type equation from an axially moving heterogeneous string modeling with boundary feedback control," Nonlinear Analysis. Theory, Methods and Applications, vol. 75, no. 8, pp. 3598-3617, 2012.

[9] D. Kim and I. H. Jung, "Asymptotic behavior of a nonlinear Kirchhoff type equation with spring boundary conditions," Computers and Mathematics with Applications, vol. 62, no. 8, pp. 3004-3014, 2011 
[10] D. Kim, Y. H. Kang, M. J. Lee, and I. H. Jung, "Energy decay rate for a quasi-linear wave equation with localized strong dissipation," Computers and Mathematics with Applications, vol. 62, no. 1, pp. 164-172, 2011.

[11] D. Kim, Y. H. Kang, J. B. Lee, G. R. Ko, and I. H. Jung, "Stabilization for a nonlinear Kirchhoff equation by boundary feedback control," Journal of Engineering Mathematics, vol. 77, pp. 197-209, 2012.

[12] I.-S. Liu and M. A. Rincon, "Effect of moving boundaries on the vibrating elastic string," Applied Numerical Mathematics, vol. 47, no. 2, pp. 159-172, 2003.

[13] F. Pellicano and F. Vestroni, "Complex dynamics of high-speed axially moving systems," Journal of Sound and Vibration, vol. 258, no. 1, pp. 31-44, 2002.

[14] S. M. Shahruz and S. A. Parasurama, "Suppression of vibration in the axially moving Kirchhoff string by boundary control," Journal of Sound and Vibration, vol. 214, no. 3, pp. 567-575, 1998.

[15] S. Bernstein, "Sur une classe d'équations fonctionelles aux dérivées partielles," Izvestiya Akademii Nauk SSSR. Seriya Matematicheskaya, vol. 4, pp. 17-26, 1940.

[16] R. W. Dickey, "The initial value problem for a nonlinear semiinfinite string," Proceedings of the Royal Society of Edinburgh, vol. 82, pp. 19-26, 1978.

[17] Y. Ebihara, L. A. Medeiros, and M. Milla Miranda, "Local solutions for a nonlinear degenerate Hyperbolic equation," Nonlinear Analysis. Theory, Methods and Applications, vol. 10, no. 1, pp. 27-40, 1986.

[18] T. G. Ha, D. Kim, and I. H. Jung, "Grobal existence and uniform decay rates for the semi-linear wave equation with damping and source terms," Computers and Mathematics with Applications, vol. 67, pp. 692-707, 2014.

[19] H. A. Levine, "Instability and nonexistence of global solutions to nonlinear equations of the form $P u_{t t}=-A u+\mathscr{F}(u)$," Transactions of the American Mathematical Society, vol. 192, pp. 1-21, 1974.

[20] J. E. Lions, "On some questions in boundary value problems of mathematical physics," in Proceedings of the International Symposium on Continuum Mechanics and Partial Differential Equations, North Holland, Amsterdam, The Netherlands, 1978.

[21] Y. Yamada, "Some nonlinear degenerate wave equations," Nonlinear Analysis, vol. 11, no. 10, pp. 1155-1168, 1987.

[22] S. I. Pohožaev, "On a class of quasilinear hyperbolic equations," Matematicheskii Sbornik, vol. 25, pp. 145-158, 1975.

[23] T. Mizumachi, "Time decay of solutions to degenerate Kirchhoff type equation," Nonlinear Analysis. Theory, Methods and Applications, vol. 33, no. 3, pp. 235-252, 1998.

[24] K. Nishihara and Y. Yamada, "On global solutions of some degenerate quasilinear hyperbolic equations with dissipative terms," Funkcialaj Ekvacioj, vol. 33, pp. 151-159, 1990.

[25] J. Y. Park, I. H. Jung, and Y. H. Kang, "Generalized quasilinear hyperbolic equations and Yosida approximations," Journal of the Australian Mathematical Society, vol. 74, no. 1, pp. 69-86, 2003.

[26] I. H. Jung and J. Choi, "Energy decay estimates for a Kirchhoff model with viscosity," Bulletin of the Korean Mathematical Society, vol. 43, no. 2, pp. 245-252, 2006.

[27] I. H. Jung and Y.-H. Lee, "Exponential decay for the solutions of wave equations of kirchhoff type with strong damping," Acta Mathematica Hungarica, vol. 92, no. 1-2, pp. 163-170, 2001.

[28] M. Ghisi and M. Gobbino, "Global existence and asymptotic behaviour for a mildly degenerate dissipative hyperbolic equation of Kirchhoff type," Asymptotic Analysis, vol. 40, no. 1, pp. 25-36, 2004.
[29] M. Aassila and D. Kaya, "On local solutions of a mildly degenerate hyperbolic equation," Journal of Mathematical Analysis and Applications, vol. 238, no. 2, pp. 418-428, 1999.

[30] S. Kouémou-Patcheu, "Global existence and exponential decay estimates for a damped quasilinear equation," Communications in Partial Differential Equations, vol. 22, no. 11-12, pp. 20072024, 1997.

[31] V. Komornik, Exact Controllability and Stabilizaion-The Multiplier Method, John Wiley, New York, NY, USA, 1994.

[32] A. Haraux, "Oscillations forcées pour certains systèmes dissipatifs nonlinéaires," Publications du Laboratoire d'Analyse Numérique, No. 78010 Université Pierre et Marie Curie, Paris, France, 1978.

[33] J. L. Lions, Quelques Méthodesde Résolution des Prolèmes aux Limites Non Linéarires, Dunod Gauthiers-Villars, Paris, France, 1968.

[34] Q. C. Nguyen and K. -S. Hong, "Simultaneous control of longitudinal and transverse vibrations of an axially moving string with velocity tracking," Journal of Sound and Vibration, vol. 331, no. 13, pp. 3006-3019, 2012. 


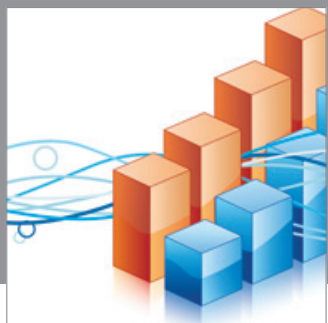

Advances in

Operations Research

mansans

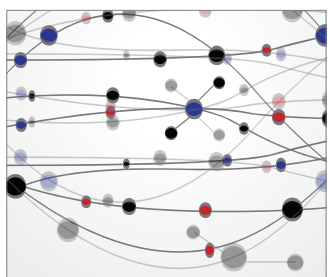

The Scientific World Journal
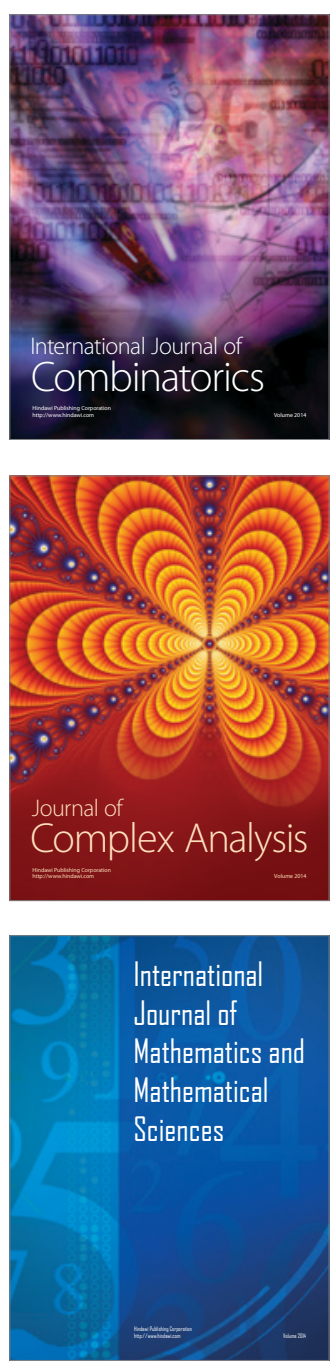
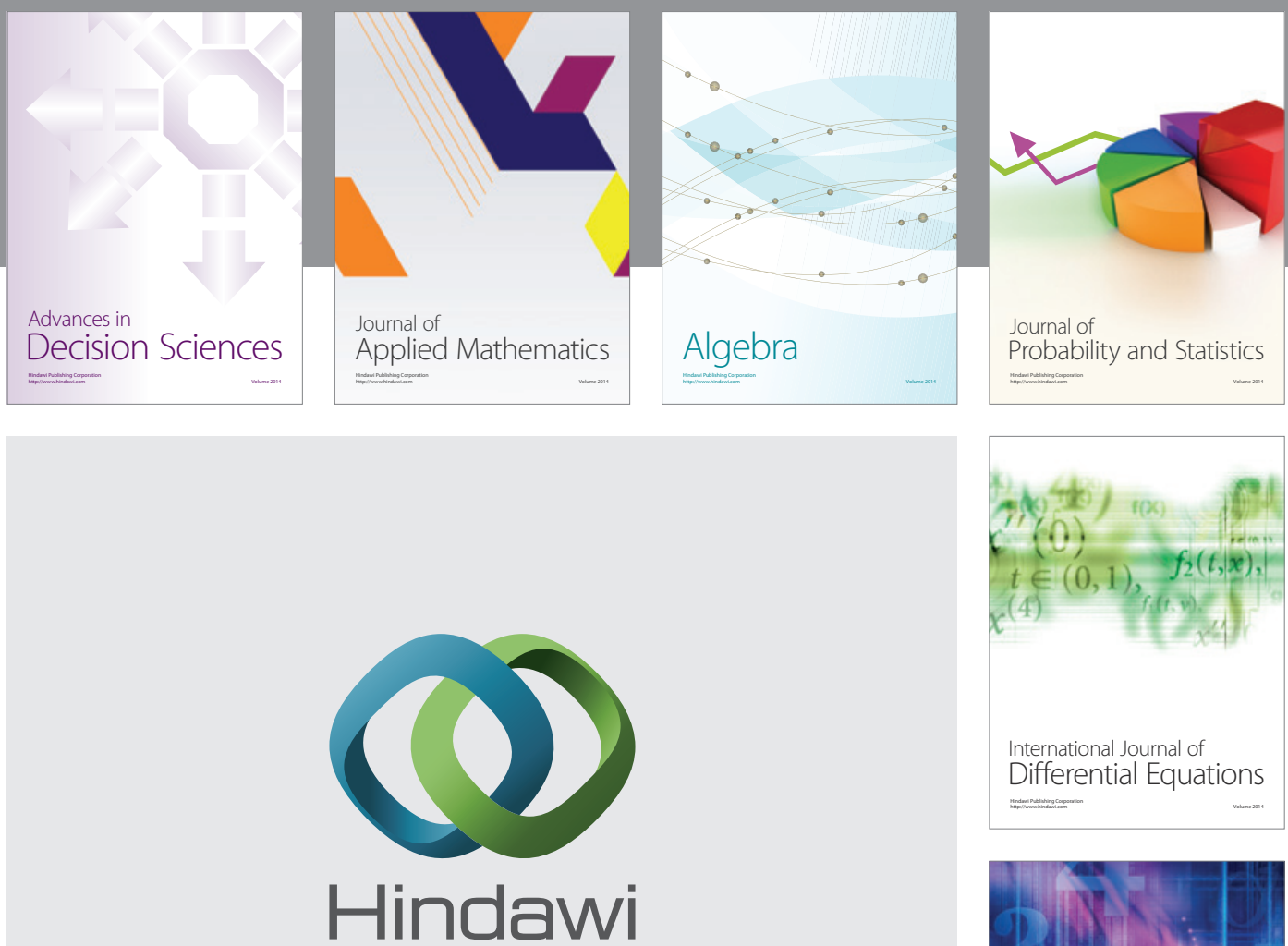

Submit your manuscripts at http://www.hindawi.com
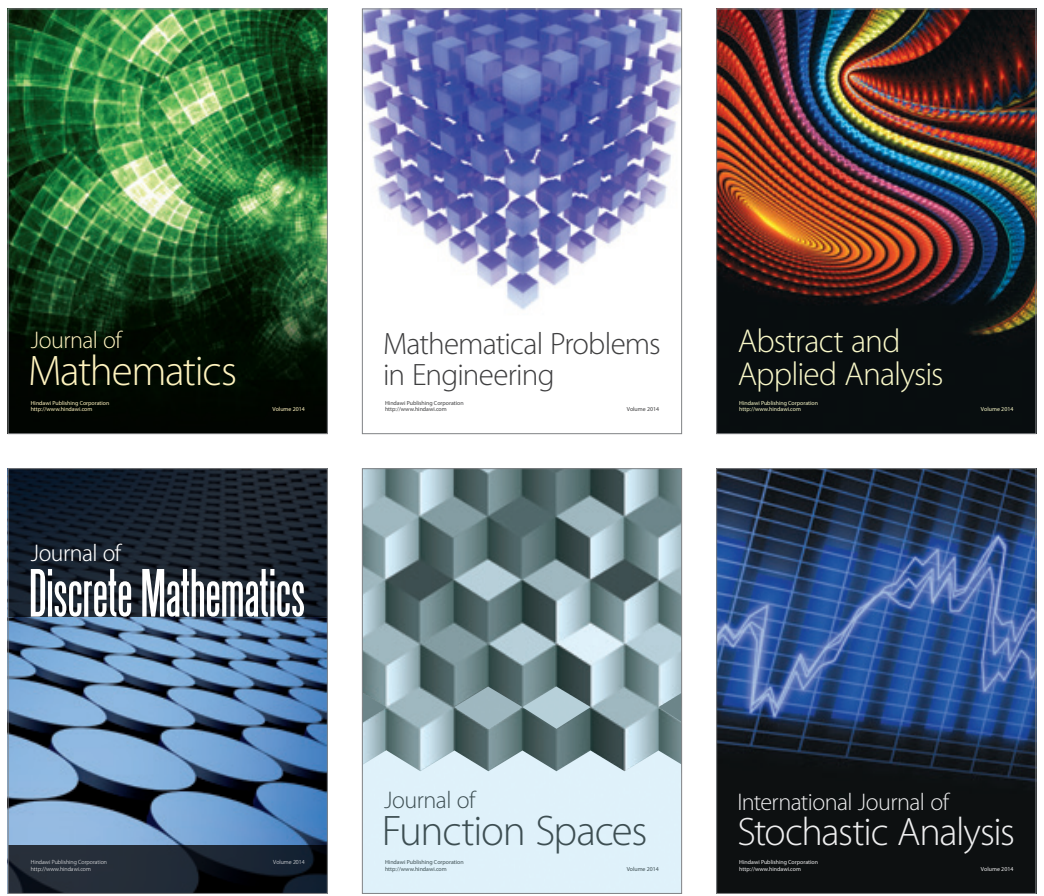

Journal of

Function Spaces

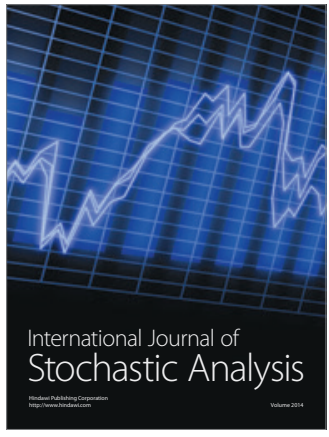

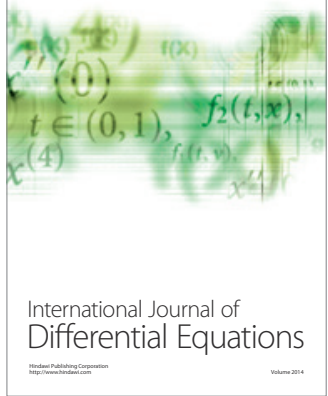
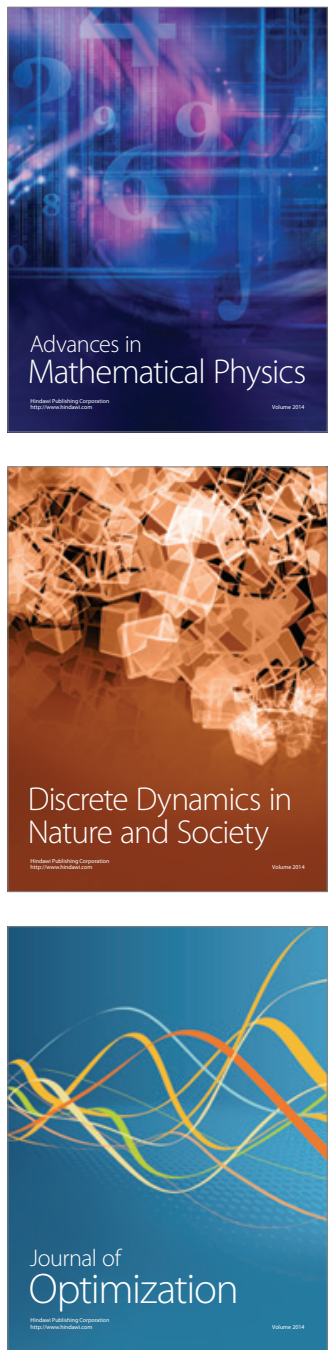